\section{Caveat on the Interpretation of Metacarpal Head Erosions Seen by Magnetic Resonance Imaging}

\section{To the Editor:}

Olech, et $a l^{1}$ recently used extremity-dedicated magnetic resonance imaging (MRI) to highlight hand lesions in patients with rheumatoid arthritis (RA) in comparison with controls. They noted that the second and third metacarpal heads were more often eroded in RA. Bone lesions, morphologically identical with erosions, were also common in controls at the same locations. A similar observation was made by Tan, et al, who linked it to anatomical causes ${ }^{2}$. The term "lesion" in MRI studies of the joints encompasses bone edema, erosions, and cysts ${ }^{3}$. According to the OMERACT definition ${ }^{4}$, an erosion is a sharply marginated bone lesion, of typical juxtaarticular localization and specific signal characteristics, which is visible on 2 planes with a cortical break in at least 1 plane. There is no definition for MRI cyst, but this could possibly correspond to lesions without cortical break, even if their location is central.

Our experience is in agreement that metacarpal heads are a common location of both erosions and cysts. Much attention should be paid to this differential diagnosis by MRI in consideration of their different prognoses. We have recently seen 3 patients with early, undifferentiated arthritis. Two men and a woman complained of metacarpophalangeal (MCP) pain for 2 to 6 months. MRI disclosed large lesions of the third MCP heads. This finding suggested a diagnosis of rapidly progressive, erosive arthritis. IgM rheumatoid factor and anticyclic citrullinated peptide (anti-CCP) antibodies were negative. Turbo 3D T1-weighed MRI showed lesions with cortical break on 3 planes and hyperintense signal on short-tau inversion recovery (STIR) sequences (Figure 1). Because of the large size of these defects, unusual for patients with early disease, computed tomography (CT) was performed to confirm.

$\mathrm{CT}$ revealed that these lesions were cysts (Figure 1). In patient A, a thin communication $(1.5 \mathrm{~mm})$ through the bone cortex was observed. Because of its small size, it was interpreted as an enlargement of a cortical bone channel $^{5}$. In the other 2 patients, no cortical disruption was seen. CT is superior to MRI in detecting bone lesions because MRI is unable to image bone per se, but can show only the bone marrow inside. Compact bone, such as that of the cyst wall of our patients, is invisible on MRI. STIR sequences, which suppress fat bone marrow, may be superior to other morphological MRI sequences for correctly interpreting cysts. In fact, compact bone, which is deprived of water and fat, appears black (Figure 1). The followup reflected our interpretation: the patients experienced disease remission after a short period of methotrexate treatment, without the onset of new bone lesions.
In general, MRI is a powerful technique to diagnose erosions because of its multiplanar imaging capability. However, in doubtful cases, careful examination of the STIR image and CT, which represents the gold standard, should be considered. Caution should be used in the diagnosis of erosions, especially in the metacarpal heads. Further prospective studies could evaluate whether cysts are a pre-erosive condition.

FRANCESCA BARBIERI, PhD; MASSIMILIANO PARODI, MD; GIUSEPPE ZAMPOGNA, MD; FRANCESCO PAPARO, MD, Diagnostic Imaging Unit, Department of Internal Medicine, University of Genoa, Genoa; MARCO A. CIMMINO, MD, Research Laboratory and Academic Unit of Clinical Rheumatology, Dipartimento di Medicina Interna, Università di Genova, Viale Benedetto XV 6, 16132 Genova, Italy Address correspondence to Prof. M.A. Cimmino;

E-mail: cimmino@unige.it

\section{REFERENCES}

1. Olech E, Crues JV 3rd, Yocum DE, Merrill JT. Bone marrow edema is the most specific finding for rheumatoid arthritis (RA) on noncontrast magnetic resonance imaging of the hands and wrists: a comparison of patients with RA and healthy controls. J Rheumatol 2010;37:265-74

2. Tan AL, Tanner SF, Conaghan PG, Radjenovic A, O'Connor P, Brown AK, et al. Role of metacarpophalangeal joint anatomic factors in the distribution of synovitis and bone erosion in early rheumatoid arthritis. Arthritis Rheum 2003;48:1214-22.

3. Ostergaard M, Peterfy C, Conaghan P, McQueen F, Bird P, Ejbjerg $\mathrm{B}$, et al. OMERACT rheumatoid arthritis magnetic resonance imaging studies. Core set of MRI acquisitions, joint pathology definitions, and the OMERACT RA-MRI scoring system. J Rheumatol 2003;30:1385-6.

4. Conaghan P, Bird P, Ejbjerg B, O'Connor P, Peterfy C, McQueen F, et al. The EULAR-OMERACT rheumatoid arthritis MRI reference image atlas: the metacarpophalangeal joints. Ann Rheum Dis 2005;64 Suppl 1:i11-21

5. Stach CM, Bäuerle M, Englbrecht M, Kronke G, Engelke K, Manger B, et al. Periarticular bone structure in rheumatoid arthritis patients and healthy individuals assessed by high-resolution computed tomography. Arthritis Rheum 2010;62:330-9.

J Rheumatol 2010;37:9; doi:10.3899/jrheum.100212 

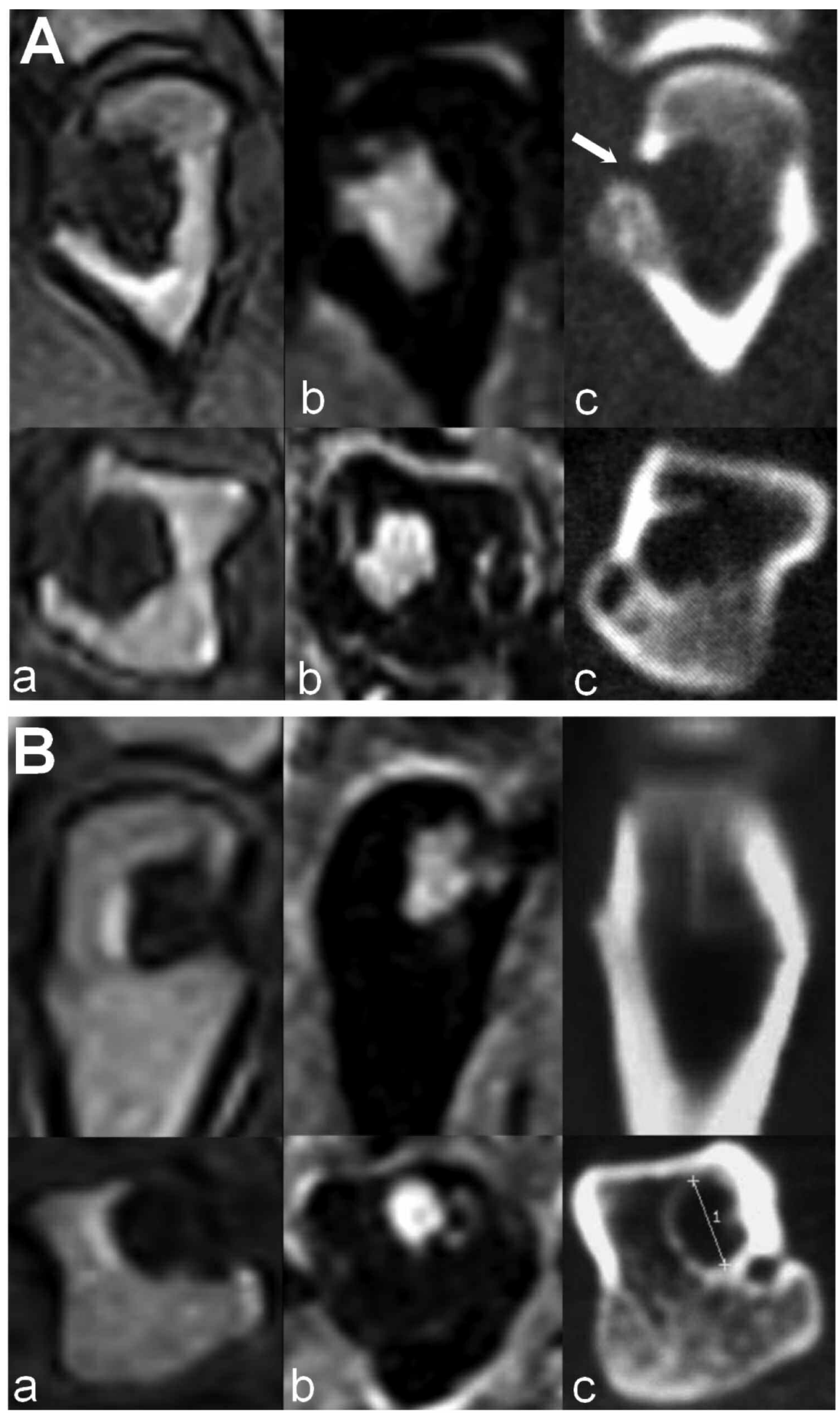

Figure 1. Imaging findings in 2 patients with early arthritis of the metacarpophalangeal joints examined by magnetic resonance imaging (MRI; a, b) and computed tomography $(\mathrm{CT} ; \mathrm{c})$. A large erosion with cortical break is seen on the MRI Turbo 3D sequence (a). On short-tau inversion recovery images (b), the cortical bone seems to be present, although faint. CT (c) clearly shows the cystic nature of the lesions, although in Patient A a thin interruption of the cortical bone is seen (arrow). The first row shows the coronal images, the second row, the axial images. 